\title{
REVIEW \\ Interaction between Torymus sinensis (Hymenoptera: Torymidae) and T. beneficus, Introduced and Indigenous Parasitoids of the Chestnut Gall Wasp Dryocosmus kuriphilus (Hymenoptera: Cynipidae)
}

\author{
Kaori YARA* \\ Biodiversity Division, National Institute for Agro-Environmental Sciences (Tsukuba, Ibaraki 305- \\ 8604, Japan)
}

\begin{abstract}
Torymus sinensis is a parasitoid wasp that was introduced from China to Japan to control the invasive chestnut gall wasp Dryocosmus kuriphilus. Interaction between T. sinensis and the indigenous parasitoid T. beneficus has been of interest since T. sinensis was first released in chestnut orchards, as hybridization between them might impede the success of biological control by the introduced parasitoid. Such apprehensions disappeared with the drastic decrease in damage caused by D. kuriphilus. However, the emergence of morphologically intermediate individuals between them after the introduction of the parasitoid has triggered renewed interest in hybridization, specifically on the non-target effects of biological control. In this review, the interaction between both species, specifically hybridization, species composition and species displacement, is surveyed. Molecular markers are used, not only because $T$. sinensis and $T$. beneficus are similar morphologically, but also because the latter has two emergence strains (early- and late-spring). Surveys show that 1) T. sinensis has displaced both emergence strains of T. beneficus; 2) hybridization between $T$. sinensis and early-spring $T$. beneficus occurs at a low frequency $(\leq 1 \%)$; but that 3 ), hybrid $\mathrm{F}_{1} \mathrm{~s}$ between $T$. sinensis and late-spring $T$. beneficus occur at a much higher frequency $(\leq 20 \%)$. A study of phylogenetic relationships indicates that the late-spring $T$. beneficus is closer to the different species $T$. sinensis than the early-spring $T$. beneficus. This can partly explain why $T$. sinensis hybridizes more readily with the late-spring $T$. beneficus than with the early-spring $T$. beneficus.

Discipline: Plant protection

Additional key words: classical biological control, displacement, hybridization, molecular marker, non-target effect
\end{abstract}

\section{Introduction}

The chestnut gall wasp Dryocosmus kuriphilus Yasumatsu, thought to have invaded from China in the 1940 s, is a serious pest affecting chestnut trees in Japan. Although the indigenous parasitoid Torymus beneficus Yasumatsu et Kamijo was experimentally released in chestnut orchards ${ }^{16}$, it was unable to suppress D. kuriphilus populations. The parasitoid Torymus sinensis Kamijo was then imported from China into Japan and released at 64 sites from 1982 to $1999^{14}$. This introduced parasitoid very effectively reduced the damage caused by $D$. kuriph$i l u s^{5,10,13}$. This project is regarded as one of the most famous and successful cases of classical biological control in Japan. However, the ecological characteristics of T. sinensis and T. beneficus remain poorly understood, partly due to the difficulty of rearing them in the laboratory and, as mentioned below, that of discriminating morphologically between them. As for T. beneficus, for example, it is not yet clear what gall insects they exploited before the invasion of D. kuriphilus in Japan; T. sinensis is thought to be a specialist parasitoid of D. kuriphilus ${ }^{9}$.

Interaction between $T$. sinensis and T. beneficus has

\footnotetext{
Present address:

Tea Research Station, NARO Institute of Vegetable and Tea Science (Shimada, Shizuoka 428-8501, Japan)

*Corresponding author: e-mail yara@affrc.go.jp

Received 27 December 2012; accepted 7 June 2013.
} 
attracted attention since the first release of $T$. sinensis in 1982: their morphological and ecological characteristics are so similar that researchers were concerned that hybridization between them might impair the effectiveness of biological control by the introduced parasitoid, e.g. through the increase in sterile individuals. Such apprehensions subsided in the face of evidence that damage by D. kuriphilus plummeted in chestnut orchards ${ }^{6}$. However, the appearance of individuals morphologically intermediate between $T$. sinensis and T. beneficus was reported in the $1990 \mathrm{~s}^{1,7}$, which sparked a new interest in hybridization, specifically the non-target effects of biological control.

Recent progress in the use of molecular markers has elicited a better understanding of insect interactions, especially hybridization, between closely-related species that are morphologically very similar. This also applies for the case of $T$. sinensis and $T$. beneficus ${ }^{17}$. Only adult females of both species can be discriminated empirically, based on a slight difference in either the length of the ovipositor or the ratio of the length of the ovipositor sheath to the thorax; but the males cannot be empirically discriminated $^{7,11,21}$. Furthermore, because $T$. beneficus has two emergence strains, namely early-spring and latespring $^{8,11}$, molecular markers could provide a more detailed insight into interactions between these two species/ strains.

In this review, I will discuss the interaction between $T$. sinensis and T. beneficus, especially hybridization, species composition and species displacement, as revealed by the use of molecular markers.

\section{Molecular markers for $T$. sinensis and both emergence strains of $T$. beneficus}

\section{Isozyme markers}

When morphologically intermediate individuals between both Torymus parasitoids appeared in the field, isozyme (or allozyme) markers were initially used to discriminate them. In $T$. sinensis and $T$. beneficus (including both emergence strains), a total of 25 isozymes were tested for available markers, only three of which (malic enzyme, adenylic acid, and aspartate aminotransferase) proved usable to discriminate between early-spring $T$. beneficus and the other parasitoid species/strain (T. sinensis and late-spring T. beneficus) 2,3 $^{3}$ (Noda et al., unpublished data). However, even these three isozymes cannot discriminate between $T$. sinensis and the late-spring $T$. beneficus. Hybridization between $T$. sinensis and the early-spring T. beneficus was therefore analyzed using an isozyme (malic enzyme), focusing on sites where the early-spring $T$. beneficus dominated.

\section{DNA markers}

Later, as replacing malic enzyme, the internal transcribed spacer 2 (ITS2) of the rRNA coding region of nuclear DNA and the cytochrome oxidase subunit I (COI) region of mtDNA were developed as discrimination markers between $T$. sinensis and $T$. beneficus ${ }^{18,20}$. Fragment analysis, which makes it possible to detect slight differences in the length of PCR products, is used as the ITS2 marker ${ }^{18}$, while PCR-RFLP is used as the COI mark$\mathrm{er}^{20}$. Both ITS2 and COI are superior to malic enzyme in their ability to discriminate, especially when used together. Neither, however, can clearly analyze hybridization of the Torymus parasitoids, since (1) the ITS2 marker cannot discriminate between some $T$. sinensis individuals and the late-spring T. beneficus; and (2) the COI marker on mtDNA is maternally inherited.

Subsequently, the internal transcribed spacer 1 (ITS1) of the rRNA coding region of nuclear DNA was developed $^{23}$. Now, three kinds of allele-specific PCR, using four designed specific primers, make it possible to distinguish Torymus species, the emergence strains of $T$. beneficus, and $\mathrm{F}_{1}$ (first filial) individuals.

\section{Hybridization between $T$. sinensis and $T$. beneficus}

\section{The early-spring $T$. beneficus}

The frequency of the malic enzyme genotype of the $\mathrm{F}_{1}$ between $T$. sinensis and the early-spring $T$. beneficus was $13 \%(n=15)$ in Tsukuba, Ibaraki Prefecture in $1992^{3}$, and $8 \%(\mathrm{n}=93)$ on the Oki Islands of Shimane Prefecture in $1996^{15}$. The $F_{1}$ genotype frequency was $1 \%(n=821)$ in specimens collected from 20 populations at 11 sites in the Tokyo Metropolis and Nagano, Ibaraki, Saitama, and Gunma prefectures from 1991 to $1995^{19}$. These studies demonstrate that hybridization had, in fact, occurred between $T$. sinensis and the early-spring $T$. beneficus, although its frequency was much lower than we expected.

Adult female specimens $(n=418)$ collected from Obuse, Nagano Prefecture during 1993-2001, were analyzed using both ITS2 and COI ${ }^{20}$. From 1993 to 1995, all specimens were the early-spring T. beneficus (Fig. 1). Torymus sinensis was first detected in 1996; the earlyspring $T$. beneficus eventually appeared to be displaced by $T$. sinensis. Simultaneously with this displacement, we found only one individual descendant of the $F_{1}$ hybrid; it had both genotypes of $T$. sinensis in ITS2 and one of the early-spring T. beneficus in COI. Hybridization between them was thus rare, and thus did not appear to be the main factor driving the displacement of the early $T$. beneficus. Torymus sinensis excels early-spring T. beneficus in reproductive ability ${ }^{12}$. 
The larvae of Torymus parasitoids $(n=448)$, collected from the same sampling site described as above (Obuse) from 1996 to 1998 and in 2000, were also analyzed using ITS2 (Fig. 2). The frequency of the $\mathrm{F}_{1}$ genotype between $T$. sinensis and the early-spring $T$. beneficus exceeded that at the adult stage, suggesting that (1) hybridization between $T$. sinensis and the early-spring $T$. beneficus did in fact occur, but that (2) most of these $\mathrm{F}_{1} \mathrm{~s}$ larvae did not survive to the adult stage.

\section{The late-spring $T$. beneficus}

From the samples above (adult female specimens (n $=418$ ) collected from Obuse, Nagano Prefecture during 1993-2001), there were also 10 individuals with both the genotype of T. sinensis in ITS2 and that of the late-spring T. beneficus in $\mathrm{COI}^{20}$. This is the first report of hybridization occurring in the field between T. sinensis and the late-spring $T$. beneficus. It also suggests that the latespring $T$. beneficus hybridized with $T$. sinensis elsewhere and that the hybrids or their descendants migrated to the research site, since originally only the early-spring $T$. beneficus had been present. These results led to research into possible interaction with late-spring $T$. beneficus. Reanalysis using ITS1 showed 49 individuals to be hybrids ( $\mathrm{F}_{1}$ and their descendants) with the late-spring $T$. beneficus (Fig. 1).

Adult female Torymus parasitoids $(\mathrm{n}=200)$, collected from Tobu, Nagano Prefecture from 1993-1996, were

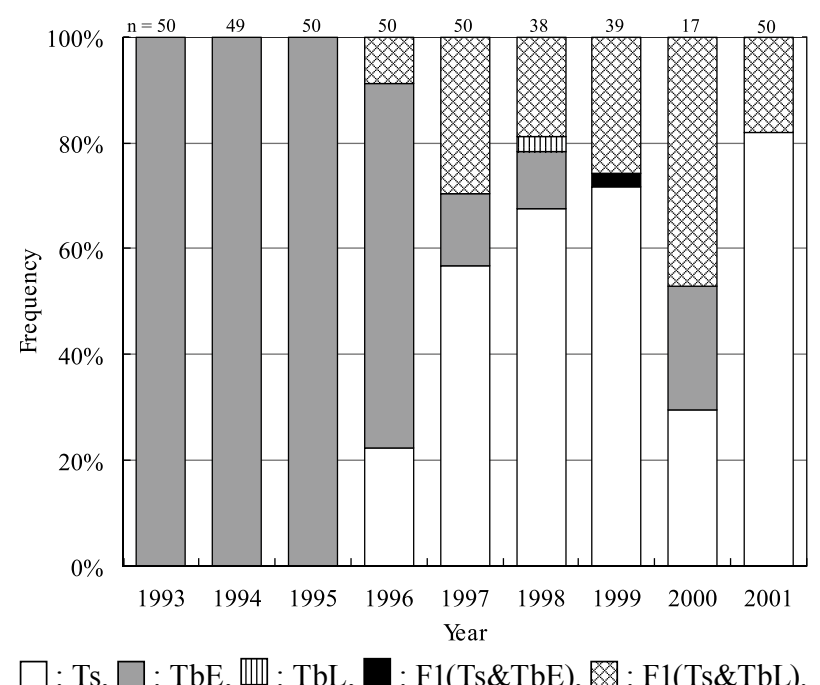

Fig. 1. Annual changes in the frequency of types of nDNA ITS2, ITS1 and mtDNA COI in adult female Torymus parasitoids in Obuse, 1993-2001

Ts: T. sinensis; TbE: early-spring T. beneficus; TbL: late-spring T. beneficus; F1(Ts\&TbE): hybrid between $T$. sinensis and early-spring $T$. beneficus; F1(Ts\&TbL): hybrid between $T$. sinensis and latespring $T$. beneficus ${ }^{20}$. analyzed using ITS1 ${ }^{21}$. Increasing numbers of $\mathrm{F}_{1}$ hybrids were detected every year from 1993 to 1996, reaching $22 \%$ (Fig. 3). It appears that $T$. sinensis can hybridize more readily with the late-spring $T$. beneficus than with the early-spring $T$. beneficus. The morphological indices of $F_{1}$ hybrids identified using the ITS1 marker ranged be-

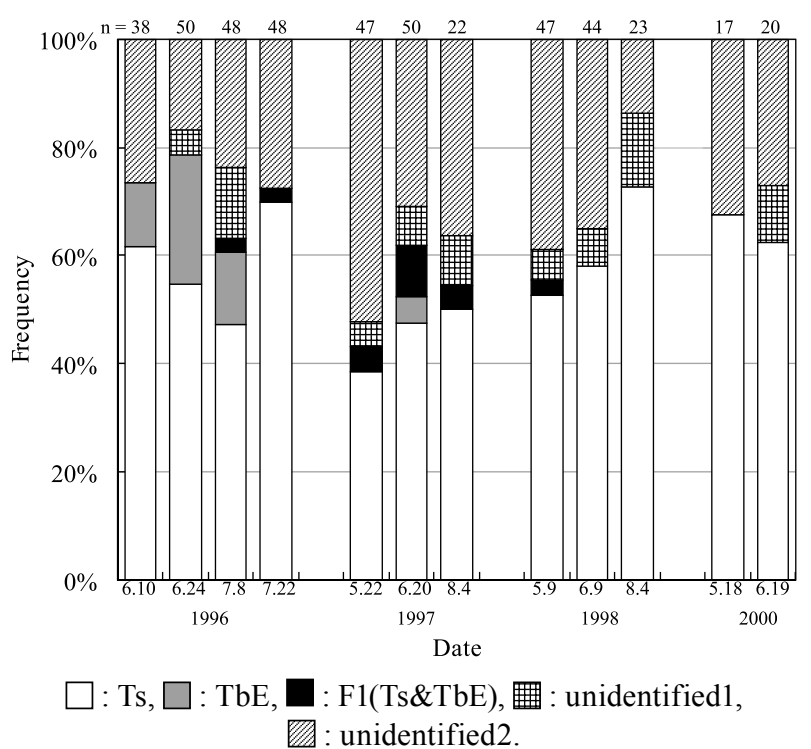

Fig. 2. Annual changes in the frequency of ITS2 type in larvae of Torymus parasitoids in Obuse, 1996-2000 Ts: T. sinensis; TbE: early-spring $T$. beneficus; F1(Ts\&TbE): hybrid between $T$. sinensis and earlyspring $T$. beneficus; unidentified 1: T. sinensis or late-spring $T$. beneficus; unidentified 2: $T$. sinensis or hybrid between $T$. sinensis and late-spring $T$. beneficus.

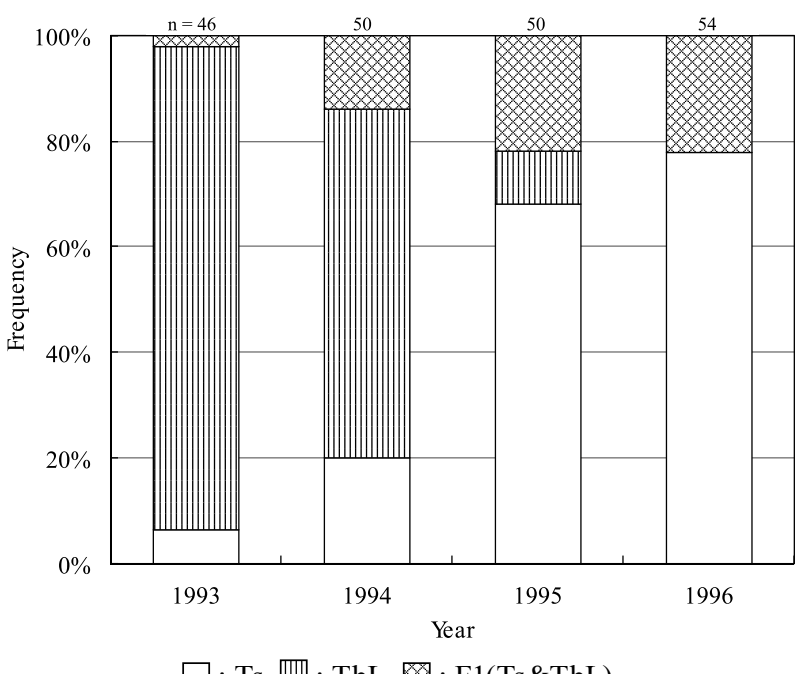

Fig. 3. Annual changes in frequency of ITS1 type in adult females of Torymus parasitoids in Tobu, 1993-1996

Ts: T. sinensis; TbL: late-spring $T$. beneficus; F1(Ts\&TbL): hybrid between $T$. sinensis and the late-spring T. beneficus ${ }^{21}$. 
tween indices of $T$. sinensis and the late-spring $T$. beneficus, with considerable overlap. Simultaneously with the occurrence of the $\mathrm{F}_{1}$ hybrids, the indigenous late-spring $T$. beneficus was rapidly displaced by $T$. sinensis. These facts suggest that hybridization is a more significant factor in the displacement of the late-spring $T$. beneficus by $T$. sinensis than in the displacement of the early-spring $T$. beneficus.

\section{Phylogenetic relationship}

A phylogenetic tree was constructed to examine the genetic relationships between $T$. sinensis and $T$. beneficus (early- and late-spring strains) collected from various places in Japan, China, and South Korea, using mtDNA COI sequences (1129bp) $)^{17}$ (Fig. 4). There were two distinct groups in the phylogenetic tree obtained: A and B. Group A consisted of two subgroups: A1 and A2 (Fig. 5). Torymus sinensis belonged to Subgroup A1 within Group A, while Subgroup A2 consisted of late-spring T. beneficus. Early-spring T. beneficus belonged to Group B. These results show that the late-spring $T$. beneficus is closer to the different species $T$. sinensis than the early-spring $T$. beneficus. This may partly explain why $T$. sinensis hybridizes more easily with the late-spring $T$. beneficus than with the early-spring T. beneficus.

\section{Further remarks}

Recent progress in the use of molecular markers has revealed complicated interactions among Torymus species/strains. For example, our study has shown their unexpected interactions in a Japanese chestnut orchard in Obuse of Nagano Prefecture where T. koreanus, endemic in Korea, was recently discovered; ${ }^{4}$ at the site, earlyspring and late-spring $T$. beneficus were almost completely displaced by $T$. sinensis for several years, whereas this was not the case for T. koreanus (Table 1). Further progress in molecular phylogenetic studies will shed light on the underlying mechanisms of the complicated interactions among Torymus species/strains and the patterns of non-target effects of classical biological control.

\section{Acknowledgements}

We wish to express our gratitude to Dr. T. Shimoda

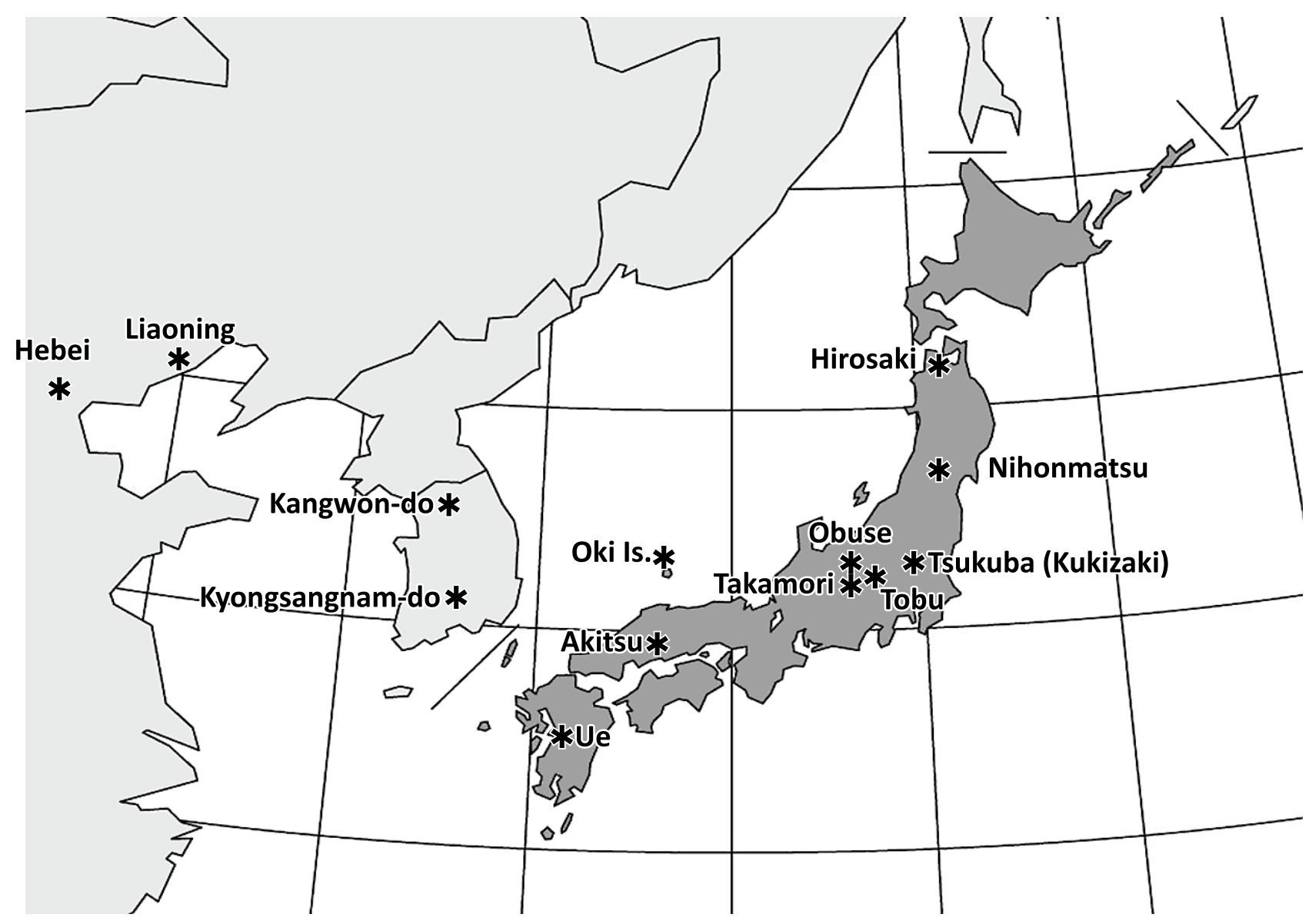

Fig. 4. Collection sites of Torymus spp. to analyze the phylogenetic relationship 
(National Agricultural Research Center) for helpful comments and suggestions on the manuscript. We appreciate both anonymous reviewers' comments that improved this article immensely. Our studies reviewed in this article were partly supported by a Grant-in-Aid (Bio Cosmos Program BCP-00-I-B-1) from the Ministry of Agriculture, Forestry and Fisheries, and a Grant-in-Aid for Scientific Research (No. 19380037) from the Japan Society for the Promotion of Science.

\section{References}

1. Aoto, I. \& Murakami, Y. (1992) Dispersion of a Torymus (Syntomaspis) sinensis population in Fukuoka Prefecture (Hymenoptera: Torymidae). Proc. Assoc. Pl. Prot. Kyushu, 38, 193-196 [In Japanese with English summary].

2. Izawa, H. et al. (1992) Isozyme discrimination between an imported parasitoid wasp, Torymus sinensis Kamijo and its sibling species, T. beneficus Yasumatsu et Kamijo (Hymenoptera: Torymidae), attacking Dryocosmus kuriphilus Ya-

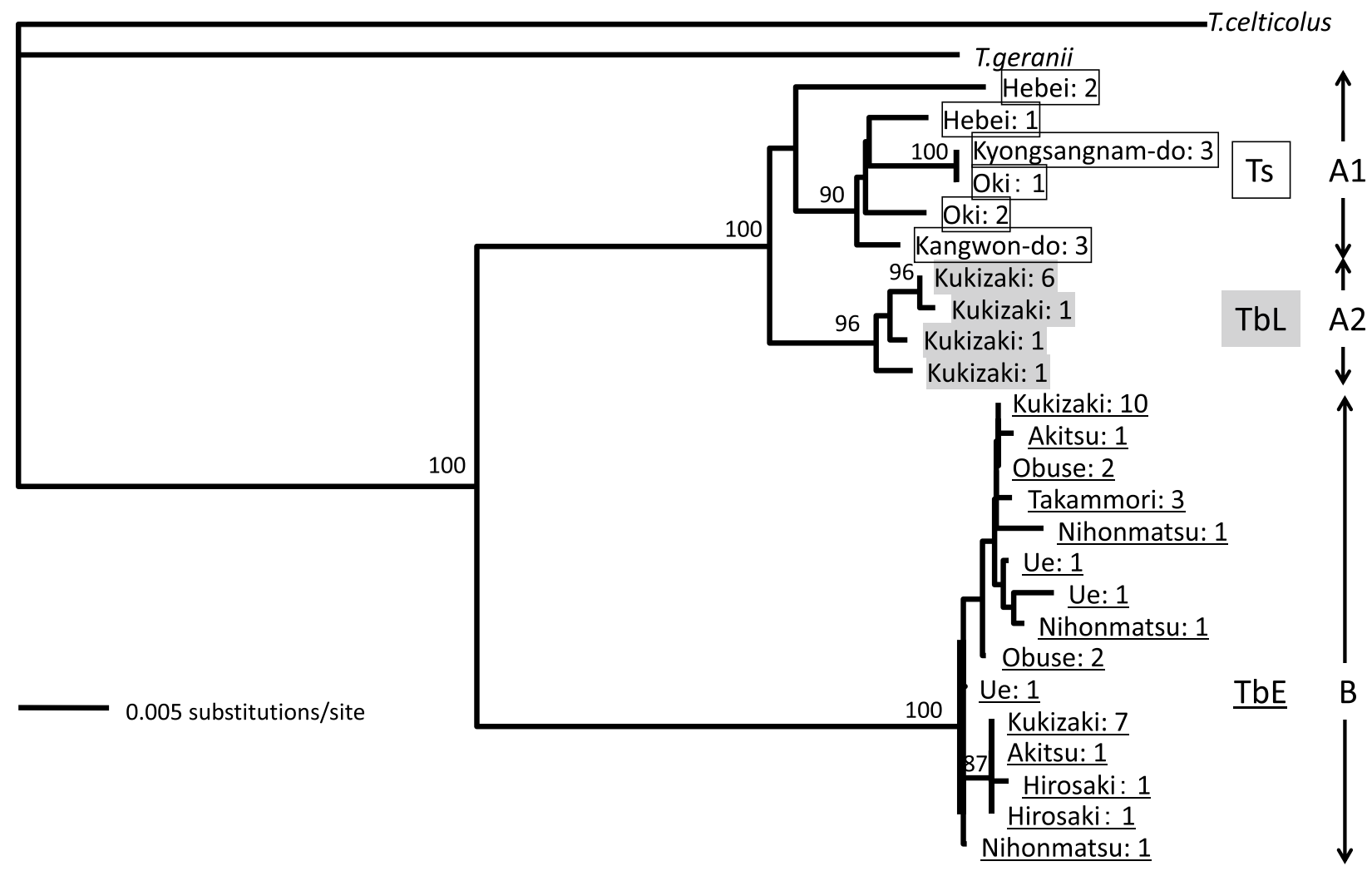

Fig. 5. Phylogenetic tree inferred from 1,129-bp sequences of the mitochondrial COI gene of Torymus spp. using the neighbor-joining method

Ts: Torymus sinensis; TbE: early-spring T. beneficus; TbL: late-spring T. beneficus. Each OTU (operational taxonomic unit) is denoted by the collection site followed by the number of specimens. Torymus geranii and T. celticolus was used as outgroups. Bootstrap probabilities (500 replicates) are provided on each branch if they exceed $70 \%$. The scale of distances is shown under the tree ${ }^{17}$.

Table 1. Composition of Torymus parasitoids collected from galls on Dryocosmus kuriphilus in Obuse $\mathrm{C}^{22}$

\begin{tabular}{|c|c|c|c|c|c|c|c|}
\hline \multirow[t]{2}{*}{ Year } & \multirow[t]{2}{*}{$\mathrm{n}$} & \multicolumn{6}{|c|}{ Torymus species ${ }^{\mathrm{a}}(\%)$} \\
\hline & & $\mathrm{Tk}$ & $\mathrm{TbE}$ & $\mathrm{TbL}$ & Ts & $\mathrm{F}_{1}(\mathrm{Ts} \& \mathrm{TbE})$ & $\mathrm{F}_{1}(\mathrm{Ts} \& \mathrm{TbL})$ \\
\hline 1992 & 24 & 20.8 & 58.3 & 20.8 & 0.0 & 0.0 & 0.0 \\
\hline 1994 & 24 & 12.5 & 54.2 & 16.7 & 8.3 & 4.1 & 4.1 \\
\hline 1996 & 24 & 16.7 & 8.3 & 0.0 & 58.3 & 0.0 & 16.7 \\
\hline
\end{tabular}

a Tk: Torymus koreanus; TbE: early-spring T. beneficus; TbL: late-spring T. beneficus; Ts: T. sinensis; $\mathrm{F}_{1}(\mathrm{Ts} \& \mathrm{TbE}): \mathrm{F}_{1}$ hybrid between $T$. sinensis and early-spring $T$. beneficus; $\mathrm{F}_{1}(\mathrm{Ts} \& \mathrm{TbL}): \mathrm{F}_{1}$ hybrid between $T$. sinensis and late-spring $T$. beneficus. 
sumatsu (Hymenoptera: Cynipidae). Jpn. J. Appl. Entomol. Zool., 36, 58-60 [In Japanese with English summary].

3. Izawa, H.et al. (1996) Use of malic enzyme to detect hybrids between Torymus sinensis and T. beneficus (Hymenoptera: Cynipidae) attacking Dryocosmus kuriphilus (Hymenoptera: Cynipidae) and possibility of natural hybridization. Jpn. J. Appl. Entomol. Zool., 40, 205-208 [In Japanese with English summary].

4. Matsuo, K. et al. (2011) Finding of Torymus koreanus (Hymenoptera: Torymidae) attacking Dryocosmus kuriphilus (Hymenoptera: Cynipidae) in Japan. Entomol. Sci., 14, 100-102.

5. Moriya, S. et al. (1989a) The use of Torymus sinensis to control chestnut gall wasp, Dryocosmus kuriphilus, in Japan. FFTC Technical Bull., 118, 1-12.

6. Moriya, S. et al. (1989b) Decline of the chestnut gall wasp population, Dryocosmus kuriphilus Yasumatsu (Hymenoptera: Cynipidae) after the establishment of Torymus sinensis Kamijo (Hymenoptera: Torymidae). Appl. Entomol. Zool., 24, 231-233.

7. Moriya, S. et al. (1992) Interspecific relationship between an introduced parasitoid, Torymus sinensis Kamijo, as a biological control agent of the chestnut gall wasp, Dryocosmus kuriphilus Yasumatsu, and an endemic parasitoid, $T$. beneficus Yasumatsu et Kamijo. Acta Phytopathologica Entomol. Hungarica, 27, 479-483.

8. Murakami, Y. (1988) Ecotypes of Torymus (Syntomaspis) beneficus Yasumatsu et Kamijo (Hymenoptera: Torymidae) with different seasonal prevalences of adult emergence. Appl. Entomol. Zool., 23, 81-87.

9. Murakami, Y. (1997) Natural Enemies of the Chestnut Gall Wasp: Approaches to Biological Control. Kyushu University Press, Fukuoka, Japan, pp.308 [In Japanese].

10. Murakami, Y. et al. (2001) Colonization by imported Torymus (Syntomaspis) sinensis Kamijo (Hymenoptera: Torymidae) of the chestnut gall wasp (Hymenoptera: Cynipidae). (7) Success in the eighteenth year after release in Kumamoto. Kyushu Pl. Prot. Res., 47,132-134 [In Japanese with English summary].

11. Ôtake, A. (1987) Comparison of some morphological characters among two strains of Torymus beneficus Yasumatsu et Kamijo and T. sinensis Kamijo (Hymenoptera: Torymidae). Appl. Entomol. Zool., 22, 600-609.

12. Piao, C. \& Moriya, S. (1992) Longevity and oviposition of Torymus sinensis Kamijo and two strains of T. beneficus Yasumatsu et Kamijo (Hymenoptera: Torymidae). Jpn. J. Appl. Entomol. Zool., 36 113- 118 [In Japanese with English summary].

13. Shiga, M. (1999) Classical biological control of the chestnut gall wasp, Dryocosmus kuriphilus: Present status and interactions between an introduced parasitoid, Torymus si- nensis, and native parasitoids. In Biological Invasions of Ecosystem by Pests and Beneficial Organisms, eds. Yano, E.et al., NIAES, Tsukuba, 175-188.

14. Shirai, Y. et al. (1999) Records of release of Torymus sinensis Kamijo (Hymenoptera: Torymidae), an introduced biological control agent of chestnut gall wasp in Japan. Bull. Natl. Inst. Fruit Tree Sci., 33, 163-178 [In Japanese with English summary].

15. Toda, S. et al. (2000) Occurrence and hybridization of two parasitoid wasps, Torymus sinensis Kamijo and T. beneficus Yasumatsu et Kamijo (Hymenoptera: Torymidae) in the Oki islands. Appl. Entomol. Zool., 35, 151-154.

16. Torii, T. (1959) Studies on the biological control of the chestnut gall wasp, Dryocosmus kuriphilus Yasumatsu (Hymenoptera, Cynipidae), with particular reference to the utilization of its indigenous natural enemies. J. Fac. Agr. Shinshu Univ., 2, 71-149.

17. Yara, K. (2004) Relationship between the introduced and indigenous parasitoids Torymus sinensis and $T$. beneficus (Hymenoptera: Torymidae) as inferred from mt-DNA (COI) sequences. Appl. Entomol. Zool., 39, 427-433.

18. Yara, K. (2006) Identification of Torymus sinensis and $T$. beneficus (Hymenoptera: Torymidae), introduced and indigenous parasitoids of the chestnut gall wasp Dryocosmus kuriphilus (Hymenoptera: Cynipidae), using the ribosomal ITS2 region. Biol. Control, 36, 15-21.

19. Yara, K. et al. (2000) Detection of hybrids between introduced Torymus sinensis and native T. beneficus (Hymenoptera: Torymidae) in central Japan, using malic enzyme. Appl. Entomol. Zool., 35, 201-206.

20. Yara, K. et al. (2007) Displacement of Torymus beneficus (Hymenoptera, Torymidae) by $T$. sinensis, an indigenous and introduced parasitoid of the chestnut gall wasp, Dryocosmus kuriphilus (Hymenoptera: Cynipidae), in Japanese chestnut fields: possible involvement in hybridization. Biol. Control, 42, 148-154.

21. Yara, K. et al. (2010) Hybridization between introduced Torymus sinensis (Hymenoptera: Torymidae) and indigenous T. beneficus (late-spring strain), parasitoids of the Asian chestnut gall wasp Dryocosmus kuriphilus (Hymenoptera: Cynipidae). Biol. Control, 54, 14-18.

22. Yara, K. et al. (2012) Influence of the introduced parasitoid Torymus sinensis (Hymenoptera: Torymidae) on T. korea$n u s$ and $T$. benefices as indigenous parasitoids of the chestnut gall wasp Dryocosmus kuriphilus (Hymenoptera: Cynipidae) on chestnut trees in Nagano Prefecture, Japan. Appl. Entomol. Zool., 47, 55-60.

23. Yara, K. \& Kunimi, Y. (2009) Discrimination of Torymus sinensis Kamijo (Hymenoptera: Torymidae) and T. beneficus Yasumatsu et Kamijo and their hybrids by allele-specific PCR. Appl. Entomol. Zool., 44, 275-280. 